\title{
Hausdorff-Based RC and IESIL Combined Positioning Algorithm for Underwater Geomagnetic Navigation
}

\author{
Yi Lin',2 \\ ${ }^{1}$ Institute of Remote Sensing and GIS, Peking University, Beijing 100871, China \\ ${ }^{2}$ Finnish Geodetic Institute, P.O. Box 15, 02431 Masala, Finland \\ Correspondence should be addressed to Yi Lin, yi.lin@fgi.fi
}

Received 1 July 2009; Accepted 21 January 2010

Academic Editor: Frank Ehlers

Copyright $(2010$ Yi Lin. This is an open access article distributed under the Creative Commons Attribution License, which permits unrestricted use, distribution, and reproduction in any medium, provided the original work is properly cited.

\begin{abstract}
This paper presents a primitive solution with novel scheme and algorithm for Underwater geoMagnetic Navigation (UMN), which now occurs as the hot-point in the research field of navigation. UMN as an independent or supplementary technique can theoretically supply accurate locations for marine vehicles, but in practice there are plenty of restrictions for UMN's application (e.g., geomagnetic daily variation). After analysis of the theoretical model of geomagnetic positioning in the correlationmatching mode from the viewpoint of pattern recognition, this paper proposed an appropriate matching scenario and a combined positioning algorithm for UMN. The subalgorithm of Hausdorff-based Relative Correlation (RC) corresponding to the pattern classification module implements the coarse positioning, and the subalgorithm of Isograms Equidistance-Segmenting the Intersection Lines (IESILs) associated with the module of feature extraction continues the fine positioning. The experiments based on the simulation platform and the real-surveyed data both validate the new algorithm, and its efficiency and accuracy are also discussed. It can be concluded that the work introduced in this paper gives an initial and real validation of UMN's potentiality.
\end{abstract}

\section{Introduction}

The technique of Underwater geoMagnetic Navigation (UMN) [1] recently has become a hot-point in the research area of navigation. This trend will develop further with the unreliability of the Global Navigation Satellite System (GNSS) increasing [2], and with the requirements growing in some special conditions (e.g., submarine exploration) [3]. It even has been deduced that only the Geophysical Field of Earth (GFE) referencing methods (UMN is a typical one) can enable Unmanned Underwater Vehicles (UUV) to navigate accurately over large areas $[4,5]$. The sea turtles using geomagnetic fields for navigation [6-9] seem to convince people more to apply UMN. Moreover, as an important supplementary means, UMN can also supply an aided solution for rectifying Inertial Navigation System's (INS's) errors which accumulate with time [10]. UMN also can be integrated with other methods [11] to improve the accuracy of marine navigation. Overall, all influences factors causing voyage drift (such as water currents or sonar failure) are taken for granted in a "black-box" in this paper, and UMN as an independent methodology will be explored to supply the accurate positioning information to rectify the whole "blackbox."

After analysis of the positioning algorithms adapted or established for UMN after the literature review, it can be summarized that batch-correlation is one kind of fundamental schemes [12-14]. The basic idea is to match the realtime sampling sequence with the picked-out geomagnetic characteristic sequences from the presurveyed maps (called for-matching sequences). The for-matching sequences are often of total intensity, and one of them is corresponding to the real-location sequence. The current locations of vehicles can be determined inversely by seeking the extreme of the correlation function, while the estimated "hunting window" (e.g., according to INS's parameters) can reduce the traversal range for extracting the for-matching sequences [15].

But the traditional positioning algorithms developed for UMN cannot let Marine geoMagnetic Field (MMF) play its full role as a good location-referencing source. Currently the often-referred correlation-type algorithms include the 
Vector Searching algorithm (VS) [12] adapted from the TERrain COntour Matching (TERCOM) algorithm originating from terrain-aided navigation [16], the improved ICP (iICP) algorithm [13] transformed from the Iterative Closest Point (ICP) algorithm initially for images registration [17], and so on. But each of these algorithms applied on MMF still suffers from some limitations. In the critical conditions which allow only one-time matching, VS even cannot supply the relatively accurate heading-angle. i-ICP is degraded by its complex matrix transformation of shift and rotation. Therefore, a more effective positioning algorithm should be built with consideration of MMF's inherent properties.

Simultaneously there are also plenty of practical restrictions hampering UMN's application (e.g., the disturbances caused by geomagnetic storm). The inherent magnetic fields of marine vehicles sometimes can locally disturb MMF, and this may make magnetometers sample the false information which is supposed to reflect MMF's real distribution. Additionally, geomagnetic fields have daily, monthly, and even annual variations. These are another kind of large influence factors to UMN and sometimes even make UMN unavailable. The improvements on hardware cannot solve all these issues. So, the positioning algorithms need some extra procedures to tolerate these undesirable factors, or even some novel and robust algorithms shall be developed from the beginning.

Consequently, from the viewpoint of pattern recognition, the theoretical models of the positioning algorithms in the matching mode are analyzed in an innovative way. Based on these analyses, this paper proposed an appropriate matching scheme and a combination positioning algorithm for UMN. The subalgorithm of Hausdorff-based Relative Correlation (RC) as the pattern classification module implements the coarse positioning and can overcome the influences of geomagnetic variations somehow. The subalgorithm of Isograms Equidistance-Segmenting the Intersection Lines (IESILs) related to the feature extraction module continues the fine positioning, which is based on the accustomed cruising-state. The state means that marine vehicles pass through the designated MMF reference areas in the Uniform Linear Motion mode (ULM) intentionally, to acquire more reliable location-associated geomagnetic information.

This paper is organized as follows. After Section 1 introducing the UMN research background, the second section makes premises analysis for a high-performance algorithm, by reviewing the traditional algorithms, generalizing UMN's restrictions, and analogizing the scenario of pattern recognition. Section 3 presents the new positioning algorithm comprising Hausdorff-based RC and IESIL. After the experimental validations in Section 4, Section 5 will go on with some discussions about the algorithm's accuracy and efficiency. Then some conclusions are achieved.

\section{Premises Analysis}

To establish a positioning algorithm appropriate for UMN with high performance, it is necessary to clarify the shortages of the traditional algorithms and to grasp the specialties of MMF. After these premises are made clear, they can work as the cut-points for a new and suitable positioning algorithm. Besides, a more proper scheme based on MMF's properties can be instructive to a more powerful algorithm. The following work in fact is an expansion of three-aspect review of traditional algorithms, analysis of MMF's specialties, and proposal for a new scheme.

2.1. Algorithms Review. From the Introduction, it can be learnt that the two typical positioning algorithms for UMN in batch-correlation mode are VS and i-ICP. Therefore, the reviews on these two algorithms can representatively give the shortages of the algorithms of this kind.

The brief idea of VS $[12,14]$ is based on the assumption of the for-matching sequences parallel with the continuously recorded trajectory (usually assuming the one indicated by INS). This simplification will introduce some errors into the matching results inherently, because INS's output of heading angle generally deviates from the real one. Continuous matching may make over this fault to some extent, but in the special conditions which urgently allow for only onetime matching or suffer from large measurement errors, the results after this method even cannot satisfy the requirement of medium-level accuracy during navigation.

The scenario of i-ICP [13] is by transforming recursively the temporary trajectory, which is connected by the projected points on the corresponding isograms, to approximate the real one. Its brief procedures contain large matrix transformation, singular value decomposition, orthogonal matrix calculation, and so on. These complex operations as well as the distortion of isograms sometimes lead to the embarrassment of solution loss. i-ICP is complicated and is not desirable for UMN under the demanding of immediate positioning, and the rasterized storage of MMF maps with large grid spacing may add the difficulties more.

From the perspective of algorithm implementation, the new algorithm for UMN needs to avoid the ill effects of parallel assumption in VS and complex transformation in $\mathrm{i}$-ICP. The omission of the yawing errors in VS can be overcome by choosing the for-matching sequences in all possible directions, and the excess of the projecting operations in i-ICP shall be reduced by restricting each formatching sequence into a line. The first and last isograms in the "hunting window" can reduce the possible directions into a reasonable extent, and marine vehicles generally can keep cruising in a line. Hereupon, modifying the positioning algorithms with some unused conditions such as cruise pattern is possible to improve their performance.

2.2. Restrictions Analysis. The factors influencing UMN remarkably include marine vehicles' own magnetic fields, the characteristic diversity of MMF maps, and the fluctuations of geomagnetic fields. The first two issues can be solved by the dragging-measurement mode and optimal-path planning separately. But for the third issue, there has been no ideal method to overcome it before.

Geomagnetic field itself has the short-time disturbances (e.g., daily variation) and the long-term fluctuations (e.g., monthly variation and even annual variation). The daily variation is illustrated in Figure 1, and long-term variation 
is exemplified in Figure 2 (a typical area with a larger magnitude of fluctuations). The daily variation makes the values of the real-time sampling sequence deviate from the presurveyed geomagnetic maps (mostly after the process of variation normalization already), with the maximum difference value up to 40 nano-Telsa (nT) indicated in Figure 1. The long-term variation makes the real-time sampled intensity away from its presurveyed maps also, with an accumulated deviation-value about $20 \mathrm{nT}$ for half a year as showed in Figure 2. Now neither can the daily variation be modeled accurately, nor can the geomagnetic variations be monitored effectively during voyage. All in all, this kind of disturbing factors can affect the positioning results with large errors and sometimes exerts the traditional correlation-based positioning algorithms fail.

In terms of the premises that samplings are limited to a certain number for each time matching, the aforementioned deviations theoretically can be simplified into a constant value. As alluded in Figure 1, the underlying basis of this simplification can be basically proved by randomly extracting time intervals with several tens of minutes, in which daily variation almost keeps constant with a very little change. Namely, the measurement sequence has a constant but valueunknown deviation compared to the for-matching sequences (also with other low-level noises). The simplification is also available for monthly and annual variations. Then the settlement is to search an effective matching criterion to judge the coincidence of the measurement sequence and the for-matching sequences, between which there is a constant difference in priority but the value of the constant is unknown. Actually, this constant-dominant difference restricts the algorithms of recursive filtering kind $[19,20]$, which have been also tried on MMFs, mostly still in the phase of methods adaptation under relatively ideal experimental environments with little noises now.

2.3. Scheme of Pattern Recognition. Sections 2.1 and 2.2 actually summarize the practical problems into two premises for an effective positioning algorithm: Premise 1 is how to assure the real-course-corresponded characteristic sequence lying in the for-matching sequences; Premise 2 is about how to choose the criterion function overcoming the influences of geomagnetic fluctuations. Consequently these require us to consider the matching process more synthetically and to seek a new scheme as the theoretical basis for a more adaptive and comprehensive algorithm.

As we know, the scheme of pattern recognition presents an efficient frame for many judgment-oriented problems. In fact, the positioning process by batch-correlation can also be classified into the research area of pattern recognition, as in how to approach the true objects (location here). Pattern recognition generally contains two-module feature extraction and pattern classification, and positioning can also be divided into these two stages. Feature extraction corresponds to the process of searching all the for-matching sequences, and pattern classification is related with the step of choosing the optimal criterion function to determine the real location.

The theoretical framework, the functional operations, and the concrete algorithms are listed in Figure 3, which

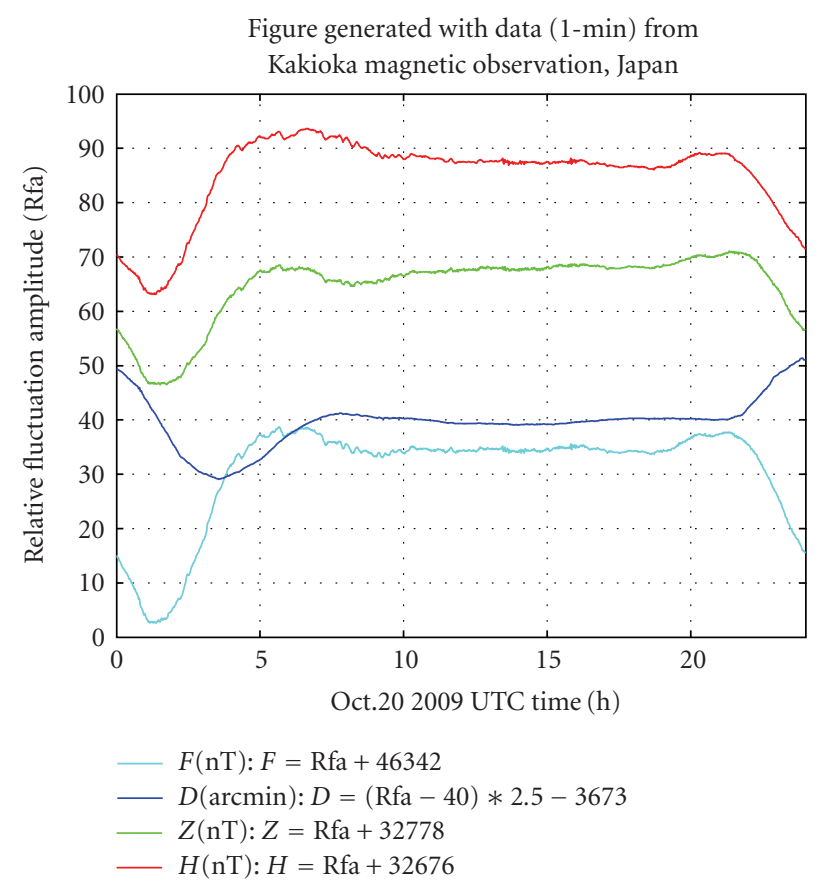

FIGURE 1: Geomagnetic variation of one day recorded by Kakioka Observatory, Japan.

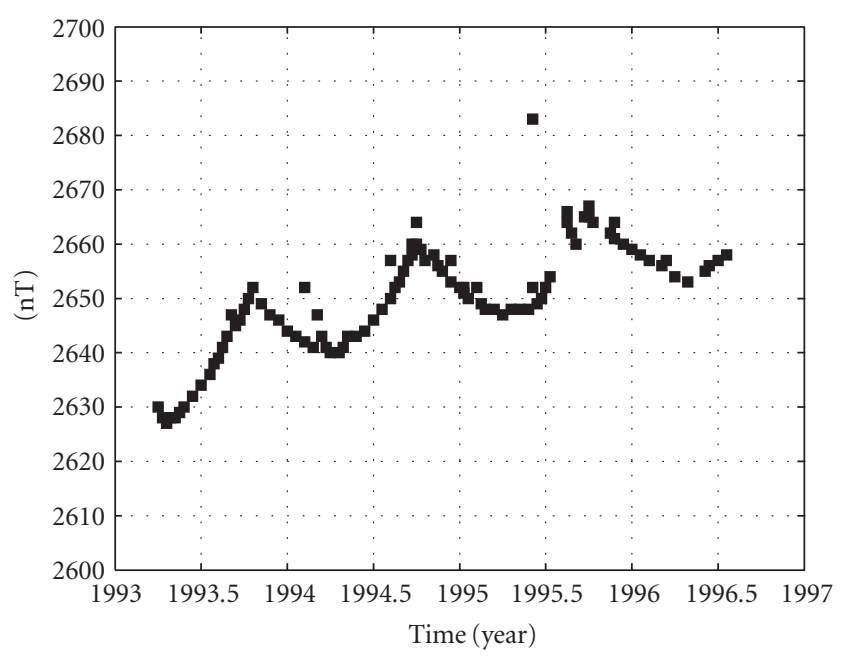

FIGURE 2: Geomagnetic long-term variation (for about three years) recorded in [18].

gives an explicit scenario of the matching process for UMN positioning algorithms. The mathematical expressions of these two steps are generalized into

$$
\begin{gathered}
\exists \tau, \tau \in\left\{\vec{x}_{i}\right\} \cap \tau \longrightarrow \vec{p}, \quad i=1,2, \ldots, N, \\
\tau=\min \left\{\left.C(\tau)\right|_{\tau=t_{i}, i=1,2, \ldots, N}\right\},
\end{gathered}
$$

where $\tau$ means the objective characteristic sequence, which corresponds to the most accurate positioning results. Function (1) denotes the necessary condition for the optimal 


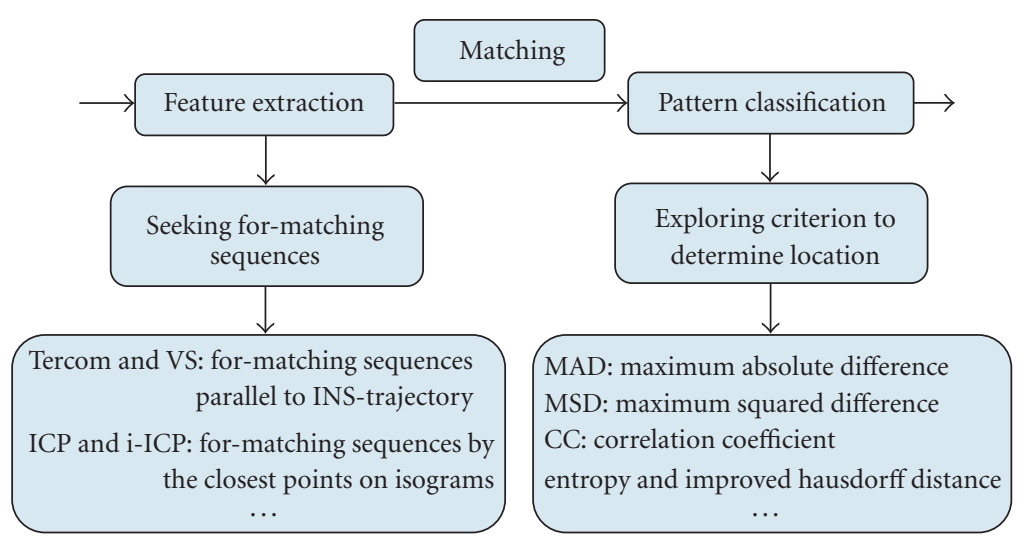

FIgURE 3: Theoretical frame of the matching process in the viewpoint of pattern recognition.

positioning algorithm, and the real trajectory must be, or close to, one of the for-matching sequences $\left\{\vec{x}_{i}\right\}$ by $\tau \rightarrow \vec{p}$. Equation (2) solves it with the goal function $C(\cdot)$.

After analysis based on this frame, it can be studied where the restrictions of the traditional matching algorithms lie. VS focuses on the module of pattern classification, while i-ICP pays more attention on the function of feature extraction. The scheme in pattern recognition gives a more effective plan, which is to improve the two modules profoundly at the same time.

Accordingly, with the scheme for matching from the viewpoint of pattern recognition displayed in Figure 3, it can be clarified that Premise 1 is involved with the module of feature extraction and Premise 2 is associated with the module of pattern classification. They are corresponding to Sections 2.1 and 2.2, respectively, and the following new algorithm is proposed by overcoming the problems mentioned in Sections 2.1 and 2.2 separately.

\section{Combination Algorithm}

The novel combination algorithm comprises two kernel subalgorithms: The subalgorithm of Hausdorff-based RC corresponds to the pattern classification module, and the subalgorithm of IESIL works as the feature extraction module. IESIL is introduced firstly, as it can somehow avoid the limitations embedded in the traditional correlation-type algorithms.

3.1. IESIL. Given the course between the two end-points of the measured sequence for positioning (named EE-course), IESIL theoretically is to approximate the ideal operation of equidistance-segmenting the EE-course. This is realized by the isograms corresponding to the real-time samplings, which are collected with equal-time intervals. Therewith, the basic scheme is as follows. Firstly, in the "hunting window" estimated with INS's parameters, the measurementscorresponded isograms are extracted; secondly, searching the intersection line with Minimum Standard Deviation (MSD) about the lengths of its line segments, which are divided out by the isograms. And then the accurate location and attitude information can be obtained inversely. All the processes are considered in the regular grid form for convenience of digital processing, and this also fits the storage mode of most MMF source data. The concrete steps are as follows.

Step 1 (Isograms extraction). As MMF maps are generally stored in grid form, tracing isograms can basically be considered just within each grid cell. Suppos that the four points $(A, B, C, D)$ comprise one little cell, in which $A$ serves as the bottom-left vertex and the other three distribute clockwise sequentially with $\left(\left(x_{1}, y_{1}\right),\left(x_{2}, y_{2}\right),\left(x_{3}, y_{3}\right),\left(x_{4}, y_{4}\right)\right)$ as their coordinates and $\left(h_{1}, h_{2}, h_{3}, h_{4}\right)$ as the characteristic values correspondingly. Then the value of any interior point $G(x, y)$ can be gained by the interpolation methods, such as bilinear interpolation with

$$
\begin{aligned}
h(G(x, y))= & (1-u)(1-v) h_{1} \\
& +u(1-v) h_{2}+(1-u) v h_{4}+u v h_{3},
\end{aligned}
$$

where $u=\left(x-x_{3}\right) /\left(x_{2}-x_{3}\right)$ and $v=\left(y-y_{1}\right) /\left(y_{2}-y_{1}\right)$. Inversely, the contour relating to a given characteristic value $c$ in cells can be expressed by

$$
\begin{gathered}
\left(h_{1}+h_{3}-h_{2}-h_{4}\right) u v+\left(h_{2}-h_{1}\right) u \\
+\left(h_{4}-h_{1}\right) v+\left(h_{1}-c\right)=0 .
\end{gathered}
$$

Equation (4) indicates that the isograms $G(x, y)$ in the cells are of hyperbolic form, which will consume a lot of computation resources. So the point set $\left\{\left(x_{i}^{n}, y_{i}^{n}\right)\right\}$ satisfying $h(G)=c$ on the four sides of each cell is alternatively searched, and then the sought $i$ points (called I-points) can be connected to construct the $n$th isograms, respectively. This simplification may introduce some little error into the positioning results but can significantly reduce computation time.

Step 2 (For-matching sequences determination]). The aforementioned for-matching sequences actually need to satisfy two conditions: (a) the whole length between the first and last isograms is equivalent to the related EE-course $D_{N}$; (b) the sequences must cross all the $N$ isograms got in Step 1. If these 
two requirements are satisfied, the associated intersectionline can be integrated into the for-matching sequences. The detailed judgment includes two parts as follows.

Firstly, the first and last crossing-points of each intersection-line are calculated. The first point assumes one I-point of the first contour determined by Step 1. Then the circle with the first point as center and $D_{N}$ as radius is constructed, and the last point can be sought as the crossing point (possibly one $\left(x_{i}^{1}, y_{i}^{1}\right)$ with several $\left.\left(x_{i_{k}}^{N}, y_{i_{k}}^{N}\right)\right)$ between the circle and the last isogram. The process can be expressed by (5). The first and last crossing points $\left\{\left(x_{i}^{1}, y_{i}^{1}\right),\left(x_{i_{k}}^{N}, y_{i_{k}}^{N}\right)\right\}$ (named EE-points) are thus determined and added into the chain list of EE-points. Then another I-point of the first isogram is picked and the same process is iterated

$$
\begin{gathered}
\left\|\left(x_{i}^{1}, y_{i}^{1}\right)^{T}-\left(x_{i_{k}}^{N}, y_{i_{k}}^{N}\right)^{T}\right\|=D_{N}, \\
\left(x_{i_{k}}^{N}-x_{j}^{N}\right) /\left(y_{j}^{N}-y_{j+1}^{N}\right)=\left(y_{i_{k}}^{N}-y_{j}^{N}\right) /\left(x_{j}^{N}-x_{j+1}^{N}\right) .
\end{gathered}
$$

Secondly, based on each pair of EE-points obtained above, the function of related intersection line can be determined, and the crossing points with the remaining isograms in middle (called M-points) can be acquired with (6). If all the remaining isograms are crossed, the EE-points and associated $\mathrm{M}$-points will be joint into one for-matching sequence. If one contour has no crossing point in the hunting window, the calculation of M-points can be stopped for the following contours, and the crossing points calculated before on this possible intersection line must be abandoned. The same operations are enforced iteratively for the whole chain list of EE-points, and the set of $M$ for-matching sequences will be generated

$$
\begin{aligned}
\left(x_{i_{k}}^{n}-x_{i}^{1}\right) /\left(y_{i}^{1}-y_{i_{k}}^{N}\right) & =\left(y_{i_{k}}^{n}-y_{i}^{1}\right) /\left(x_{i}^{1}-x_{i_{k}}^{N}\right), \\
\left(x_{i_{k}}^{n}-x_{j}^{n}\right) /\left(y_{j}^{n}-y_{j+1}^{n}\right) & =\left(y_{i_{k}}^{n}-y_{j}^{n}\right) /\left(x_{j}^{n}-x_{j+1}^{n}\right) .
\end{aligned}
$$

Step 3 (Minimum MSD calculation]). Seeking the minimum MSD about the lengths of the line segments, which is based on (7), is the concrete mathematical implementation of the isograms equidistance-segmenting the intersection lines. The location $\left(x_{i_{k}}^{N}, y_{i_{k}}^{N}\right)$ relating to the minimum MSD will be the most possible positioning result. The heading angle $\theta$ can also be solved by $\tan \theta=\left(y_{i_{k}}^{N}-y_{i}^{1}\right) /\left(x_{i_{k}}^{N}-x_{i}^{1}\right)$, and $\theta$ will be valued with $90^{\circ}$ under the special condition $x_{i_{k}}^{N}=x_{i}^{1}$

$$
\begin{aligned}
& \operatorname{MSD}\left(G\left(x_{i}^{n}, y_{i}^{n}\right)\right) \\
& =\min \left\{\sum_{n=1}^{N-1}\left(\sqrt{\left(x_{i+1}^{n}-x_{i}^{n}\right)^{2}+\left(y_{i+1}^{n}-y_{i}^{n}\right)^{2}}-\frac{D_{N}}{N-1}\right)^{2}\right\}_{1 \leq i \leq M} .
\end{aligned}
$$

Step 4 (Recompare iteratively with half-spacing]). After the intersection line with the minimum MSD is achieved, the two points $P$ and $Q$ with distance of half grid interval to the first point $O$ on the same contour are picked out. Then Steps 2 and 3 are iterated on these two points. (A) If the minimum
MSD related with points $P$ and $Q$ is larger than $O$, the point $O$ can be determined as the real position. (B) Otherwise, $P$ or $Q$ with less MSD replaces $O$, and the STEPs are iterated from $P$ or $Q$ until the condition (A) is satisfied. The heading angle can be resolved. In case of excessive iteration, the number of times for approximating the new first point by $P$ and $Q$ is restricted with a given threshold value.

The four steps depicted above can ultimately output the solved location $\left(x_{i}^{n}, y_{i}^{n}\right)$ and heading angle $\theta$ of marine vehicles.

3.2. Hausdorff-Based RC. IESIL can somehow diminish the issues listed in Section 2.1. But for the restrictions in Section 2.2, IESIL cannot overcome those problems just by improving the feature extraction module. The deviation of the positioning results caused by geomagnetic fluctuations shall be reduced in the module of pattern classification by exploring MMF's properties.

This subsection explains RC with the traditional criteria, such as Mean Absolute Difference (MAD) and Mean Square Difference (MSD) in (8). It is theoretically proper for MMFs. The idea of $\mathrm{RC}$ is to subtract the corresponding mean values from all for-matching sequences and then calculate the correlation degree between them and the measurement sequence also with its average removed. With this preprocess, the constant-like deviation of real-time samplings caused by daily variation or other variations can be omitted during the matching. As matching for each time takes just a certain number of samplings, both averages removal can overcome the influences of daily variation and so on

$$
\begin{aligned}
\operatorname{RC}_{\mathrm{MAD}} & =\operatorname{MAD}\left(T_{R}, D_{R}\right) \\
& =\frac{1}{V} \int|[T(x, y, z)-\bar{T}]-[D(x, y, z)-\bar{D}]| d v, \\
\mathrm{RC}_{\mathrm{MSD}} & =\operatorname{MSD}\left(T_{R}, D_{R}\right) \\
& =\frac{1}{V} \int\{[T(x, y, z)-\bar{T}]-[D(x, y, z)-\bar{D}]\}^{2} d v
\end{aligned}
$$

where $T_{R}$ and $D_{R}$ are the measurement sequence and formatching sequence with their averages $\bar{T}$ and $\bar{D}$ removed. The minimum correlation results give the real trajectory, and the new judging function with the improved criterion of Hausdorff distance is formulated into

$$
\begin{aligned}
\mathrm{RC}_{\text {Hausdorff }} & =H\left(T_{R}, D_{R}\right) \\
& =\max \left(\max d\left(T_{i}-\bar{T}\right), \max d^{\prime}\left(D_{i}-\bar{D}\right),\right),
\end{aligned}
$$

where $H(A, B)=\max \left(\max d_{a \in A}(a), \max d^{\prime}{ }_{b \in B}(b)\right.$, ) with the extreme-searching subfunctions of $d(x)=\min _{b \in B}\|x-b\|$ and $d^{\prime}(x)=\min _{a \in A}\|x-a\|$.

Hausdorff distance reveals the mismatch degree between the characteristic sets $A$ and $B$. Namely, a larger Hausdorff distance denotes the greater difference between two characteristic sequences. Its advantage is insensitive to low-level noises. Thus, with the improvements of Hausdorff distance 


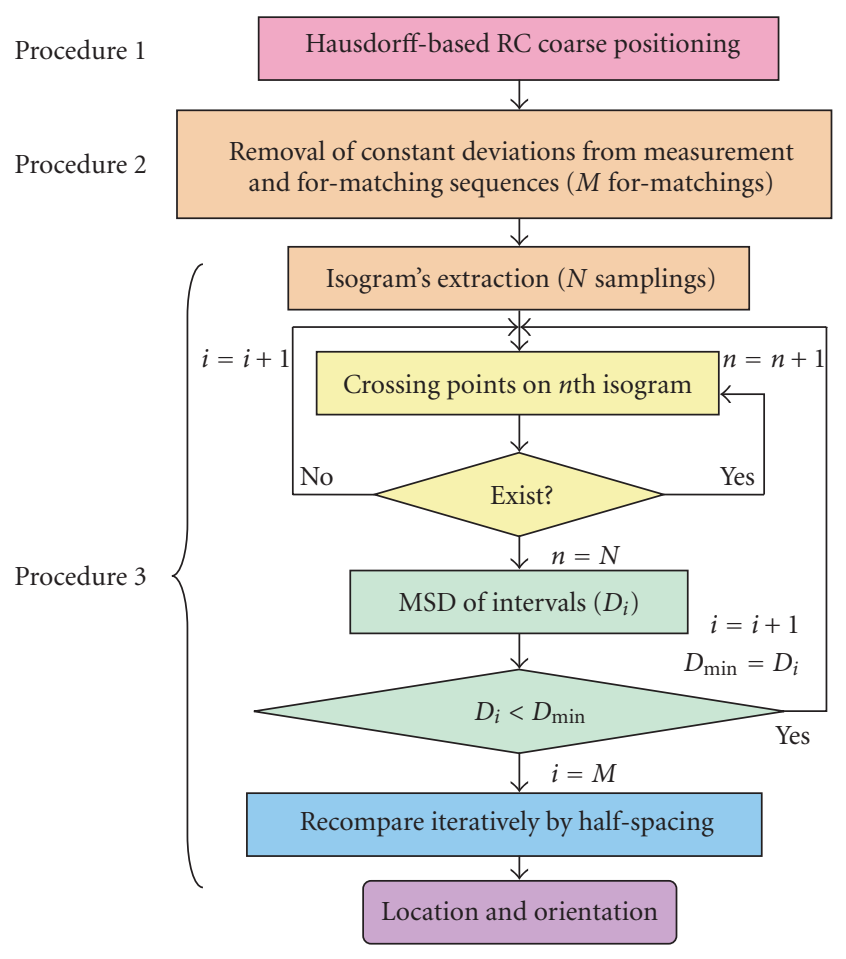

FIGURE 4: Workflow of the combination algorithm for UMN.

to overcome the measurement random noises and $\mathrm{RC}$ to reduce the whole deviation produced by geomagnetic fluctuations, the subalgorithm of Hausdorff-based RC supplies a good settlement to MMF's own disturbances.

3.3. Procedures. Based on the two subalgorithms established above, this subsection presents a full scenario of the proposed positioning algorithm. Hausdorff-based RC is carried out for coarse positioning, and then IESIL is deployed for further fine positioning. The procedures of the combination algorithm in Figure 4 are depicted in detail as follows.

Procedure 1. The set of for-matching sequences parallel to the trajectory indicated by INS are extracted, and Hausdorffbased RC is run based on this sequence set for coarse positioning.

Procedure 2. With the results of coarse positioning, the constant-in-priority deviations caused by the influences (e.g., daily variation) can be rectified from the measurement sequences.

Procedure 3. Based on the results of the coarse positioning and the measurement sequences after rectification, IESIL completes the fine positioning. The process takes the coarse location as the center of the hunting window, and the real location can be achieved iteratively.

\section{Experiments}

The experiments are firstly conducted on our simulation platform to testify the new algorithm's applicability to the

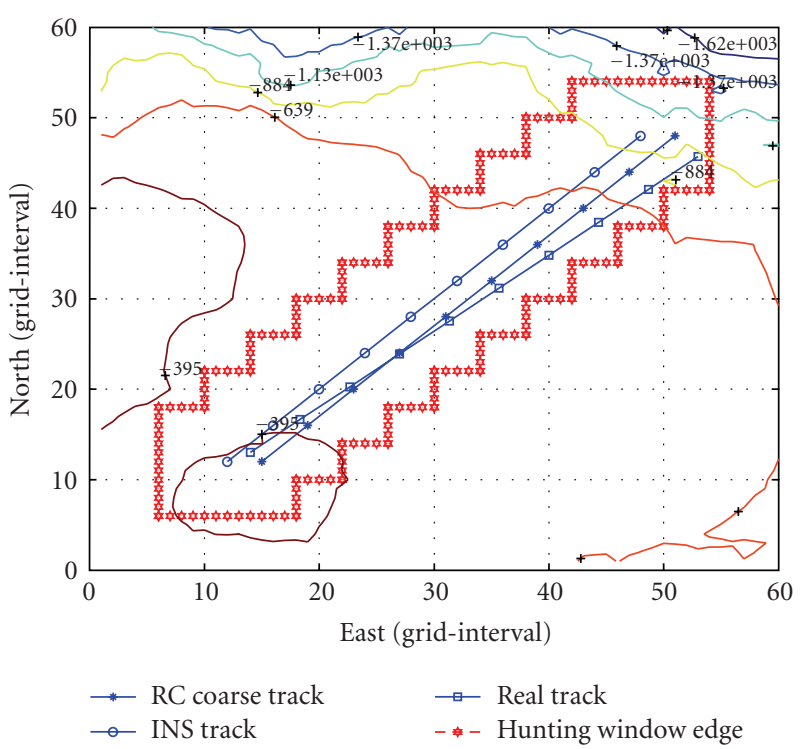

(a)

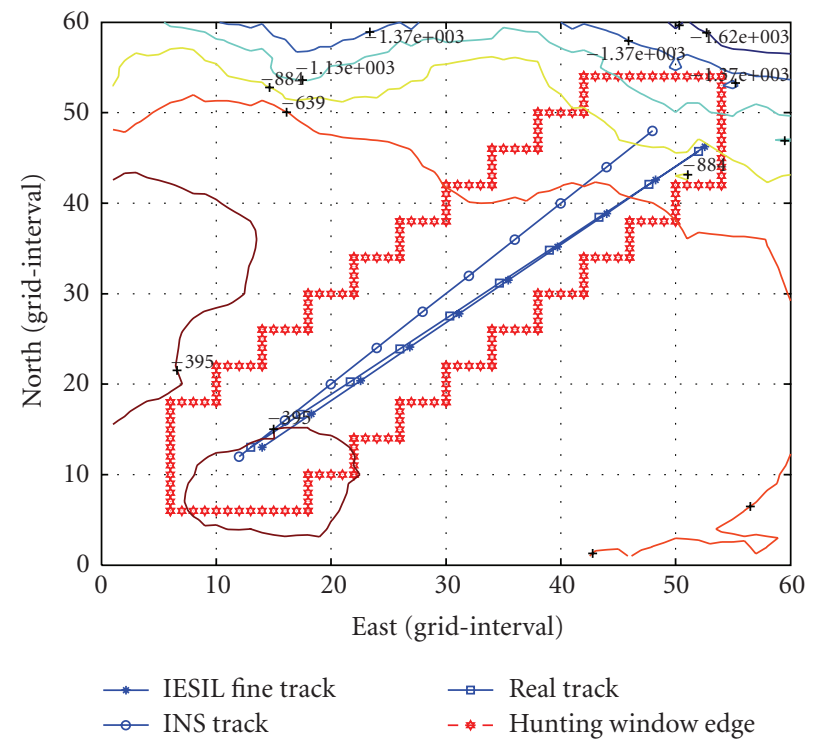

(b)

Figure 5: Results of simulation experiments: (a) RC coarsepositioning, (b) IESIL fine-positioning.

diversity of the geomagnetic reference maps, and some experiments are also tried on the real-surveyed MMF data to validate the algorithm in real environments.

4.1. Simulation Experiments. The simulation experiments were executed on some local geomagnetic maps. The positioning results in Figure 5 give an illustration of the positioning accuracy of the combination algorithm. The related MMF map is stored in grid form with $60 \times 60$ points and is collected in geomagnetic total intensity. The compositional anomaly intensity is between 1.4 and $265.2 \mathrm{nT}$. The display module assumes the grid spacing as the coordinate unit (named grid-interval). 
The experimental conditions were configured as follows. The initial location indicated by INS is $(12,12)$, and the yawing angle is $45^{\circ}$ to north. The distance between two samplings is 5.7 grid-intervals, and the sampling number accumulates to 10 then to comprise a for-matching sequence. The real start point lies in the circle with radius of 2 gridintervals and with center at the start location indicated by INS. The real heading angle is $50^{\circ}$ to north, and the sampling interval is equal to INS's one. The errors of samplings are yielded randomly as Gaussian white noises with the standard deviation of $5 \mathrm{nT}$. The daily variation is simulated with a deviation of $20 \mathrm{nT}$, which is added to the measurement sequence.

The result of coarse positioning with Hausdorff-based $\mathrm{RC}$ is showed in Figure 5(a). It indicates that the resulted trajectory is still parallel with the output one by INS. And the yawing error remains nonignorable. Next, the measurement sequence is modified by setting its initial value equal with the first one of the resulted sequence after coarse positioning. Then IESIL is executed and the result of fine positioning is demonstrated in Figure 5(b), and it approximates the real trajectory with deviation less than 1 grid interval. The results of Hausdorff-based RC and IESIL both lie in the "hunting window". From Figure 5(a) to Figure 5(b), the validity of the combination algorithm can be explicitly judged by the spatial relationships between the coarsely resulted track, fine-resulted track, INS track, and real track. IESIL-resulted fine track is more close to the real track than to RCresulted coarse track, and both are better than the INS track. The rectifying effect for INS's drift is very obvious. The comparison basically confirms the anticipation that the combination algorithm can supply a more effective method for UMN.

4.2. Real Data Validation. The disturbances in practical environments are more complex than the assumptions of noises in the simulation platform. To validate the combination algorithm, the postprocessing experiments based on the real MMF data are executed. The surveying of the MMF data was accomplished with recording of INS and GPS data synchronously at Bo Sea from June 23, 2008 to August 17, 2008.

4.2.1. Real-Surveyed Data. The surveying conditions at Bo Sea were planed as such the region occupies 10 kilometers in the west-east direction and 5 kilometers in the north-south direction. The range of geomagnetic total intensity is (53476, 53642) nT. The location on Google-Earth is indicated in Figure 6.

The magnetometer assumed is Ocean G882 of cesium optical-pump type. Its sampling frequency is set with $5 \mathrm{~Hz}$, and its measurement resolution is adapted to $0.001 \mathrm{nT}$. INS uses the Laser INS with the moderate drift of $0.01 \%$ h. GPS applies the Trimble-type Difference GPS (DGPS) with precision of $0.1 \mathrm{~m}$. The ship's velocity is $5-8$ knots (1 knot $=1.852$ kilometers/hour). The distance between the measurement lines is $200 \mathrm{~m}$, and the sampling points on each line have the average interval of $0.1 \mathrm{~m}$. The boat is mainly of wood as displayed in Figure 7, but it still has about 4 tons

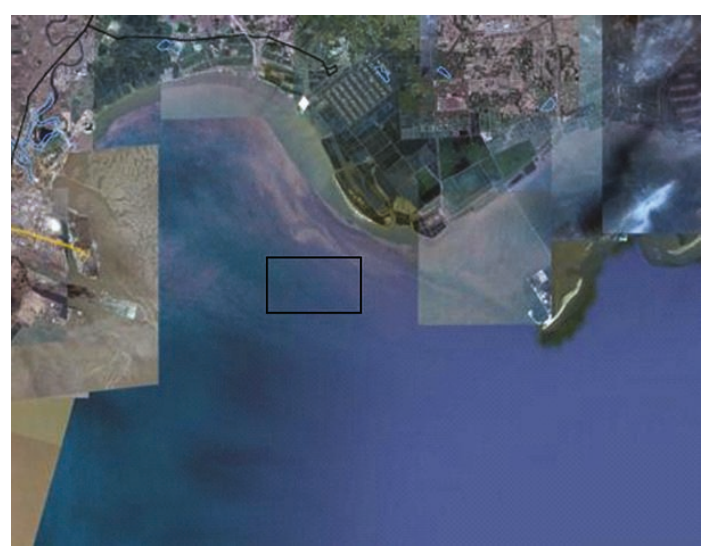

Figure 6: The area at Bo Sea for geomagnetic surveying.

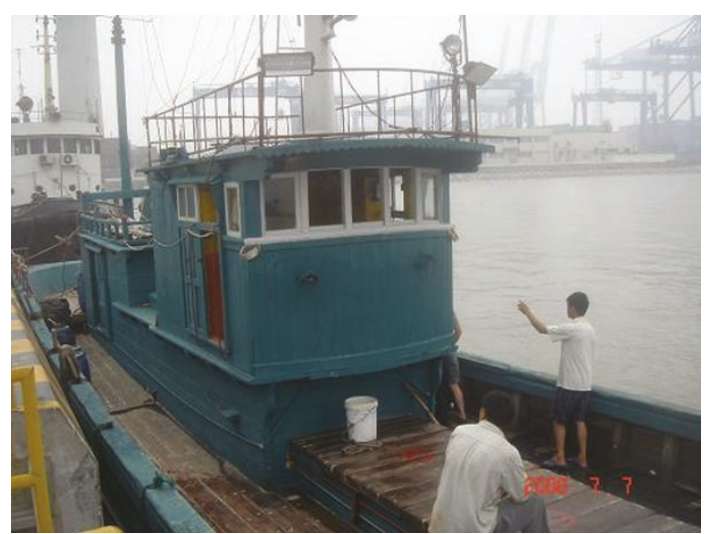

FIGURE 7: The wood-dominant ship for geomagnetic surveying.

of iron materials. So the dragging-measurement mode with cable length of $50 \mathrm{~m}$ is utilized, and the magnetometer floats between the sea-surface and $2 \mathrm{~m}$ below. If a metal-dominant boat uses UMN, maybe a longer cable and a more precise dragging-model are needed.

The local MMF map constructed from the surveyed data is presented with isograms in Figure 8. A certain number of samplings from the diagonal course, which is designed for testing, were picked out as the measurement sequence. The voyage direction is from south-east to north-west. The GPSindicated and INS-indicated trajectories are drawn also in Figure 8, and they are chosen on purpose to represent the situation with great INS's drift. Under the relative reference coordinates with (North, East) as the variable, the initial point of GPS-indicated trajectory lies in $(32.3088,58.3222)$, and the initial point of INS-indicated trajectory lies in (38.5096, 42.4688). Their last points have locations (43.1052, $37.2662)$ and $(49.3948,21.6530)$ individually.

4.2.2. Data Analysis. The statistical analysis of this MMF area manifests that the range of the geomagnetic intensity is $(53476,53642) \mathrm{nT}$, and its standard deviation is 43.49 . The standard deviation suggests that this area statistically has ample geomagnetic fluctuations suitable for UMN, but the distribution trend of geomagnetic intensity is declining 


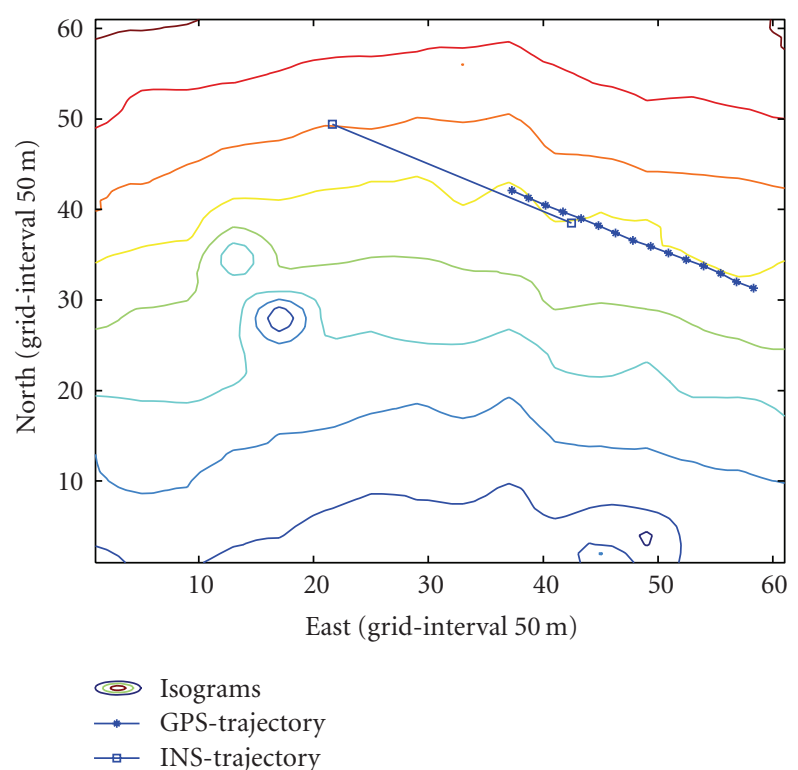

FIGURE 8: Geomagnetic map in local reference coordinates and the trajectories recorded by INS and GPS.

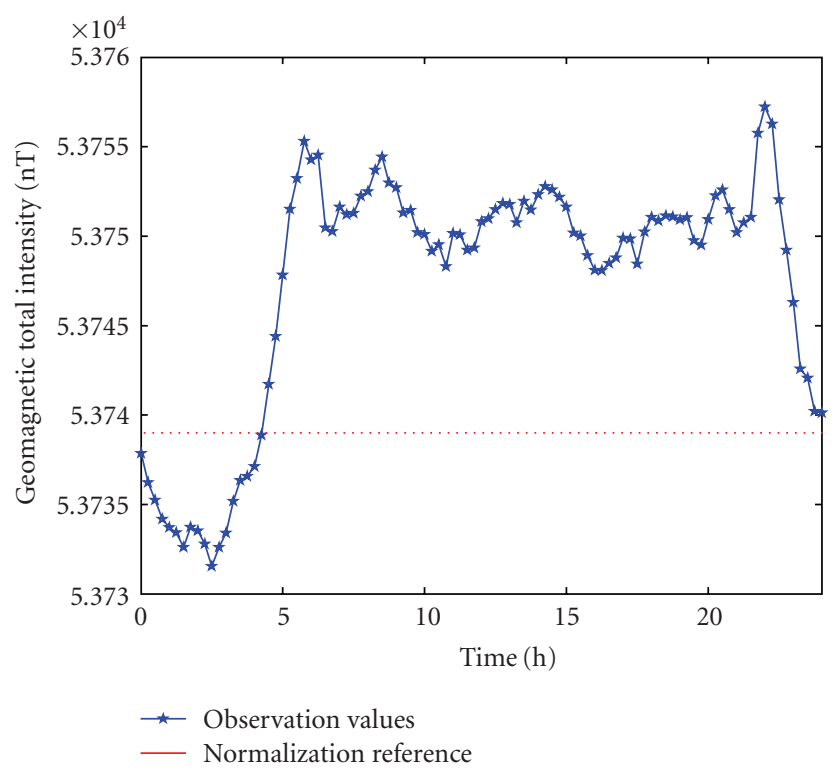

FIGURE 9: Daily variation corresponding to the real-measurement sequence.

south-to-north in a relatively smooth mode. This indicates that the area is not an ideal region with rich spatial referencing characteristics, but for validating the positioning algorithm it can supply a stringent environment.

As showed in Figure 8, the heading bias between INStrajectory and GPS-trajectory is small, but the location deviation is large. The distance is $851 \mathrm{~m}$ from the last location output by INS to the one indicated by DGPS. This indicates that INS has a great drift and it needs the rectification by UMN. The location sequence from DGPS, which delegates

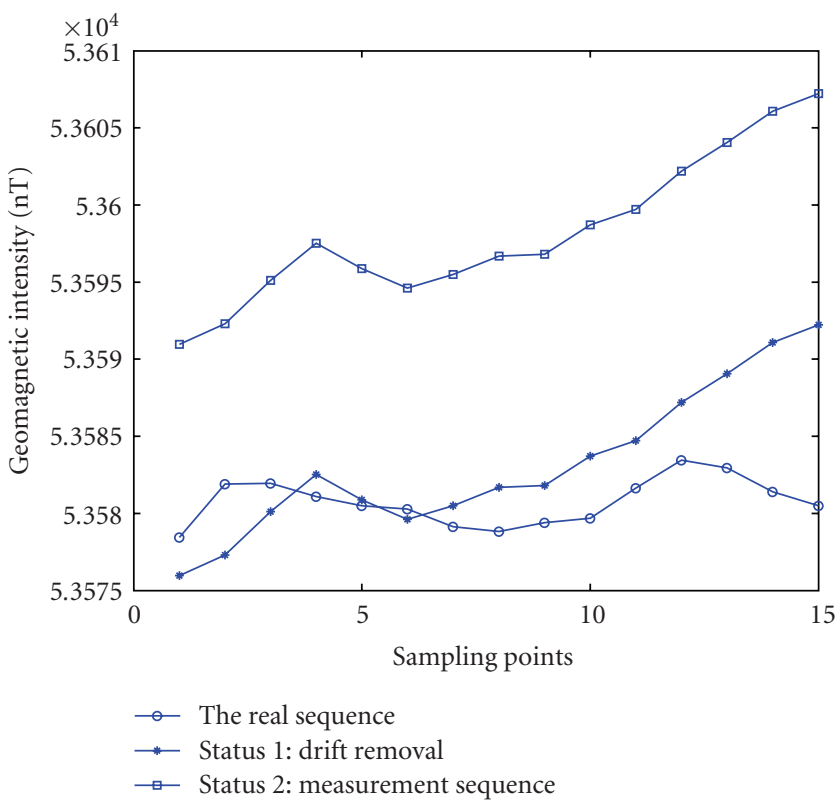

FIGURE 10: Comparison between the real-measurement sequence and the processed sequence with a constant difference removed for cross-validation.

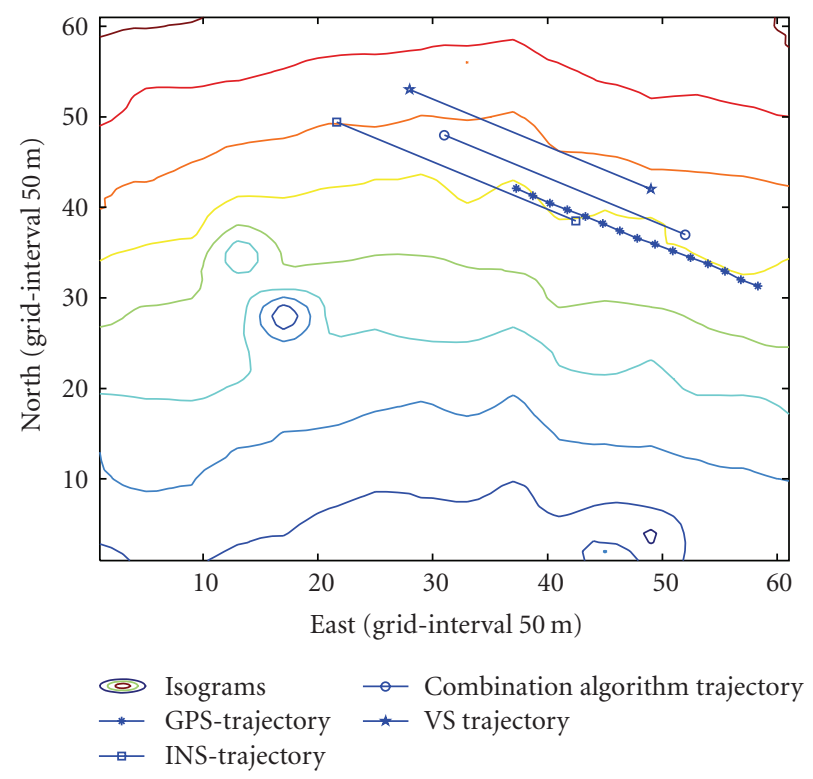

Figure 11: Results after the combination algorithm and the traditional algorithm.

the track of the vehicle, is linear on the whole, and this means that the boat cruises in a line. As a result, the realsurveyed data can be used as the references for validating of the combination algorithm.

The geomagnetic reference map is the result after normalization of daily variation, while the real-time measurement sequence during navigation still keeps this influence factor. The daily variation corresponding to the real-measurement is segmented out and demonstrated in Figure 9. The normalization value is related with the dotted 


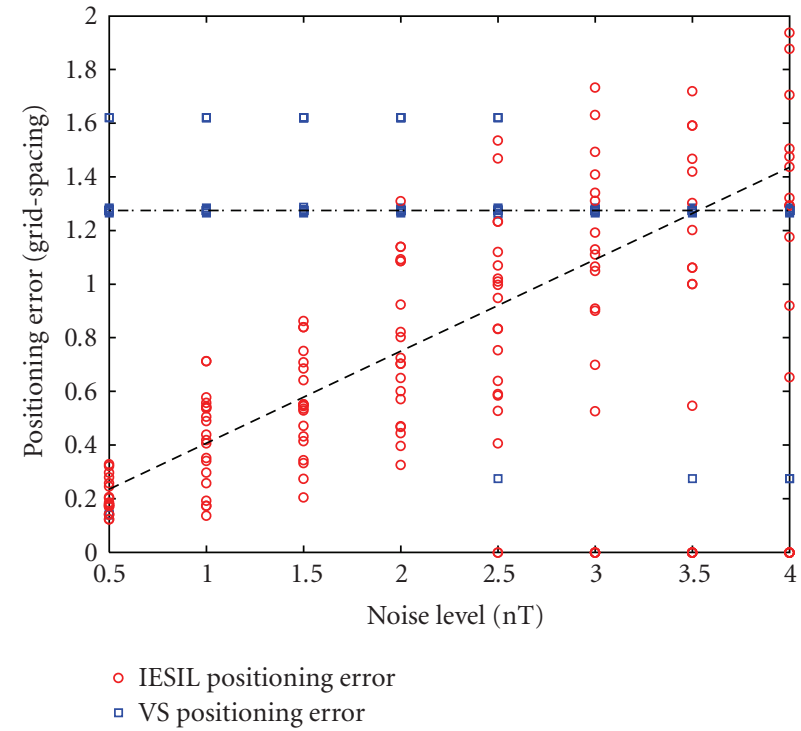

(a)

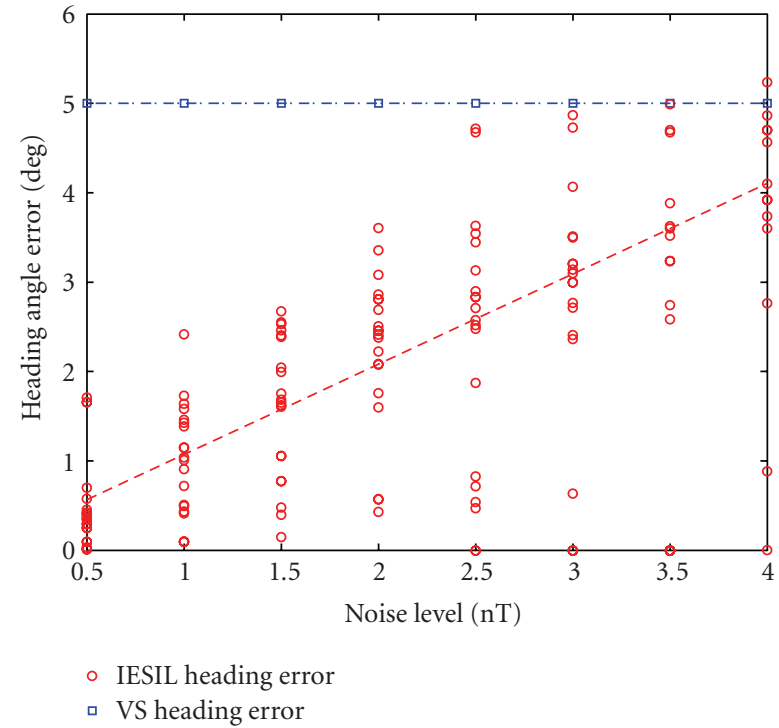

(b)

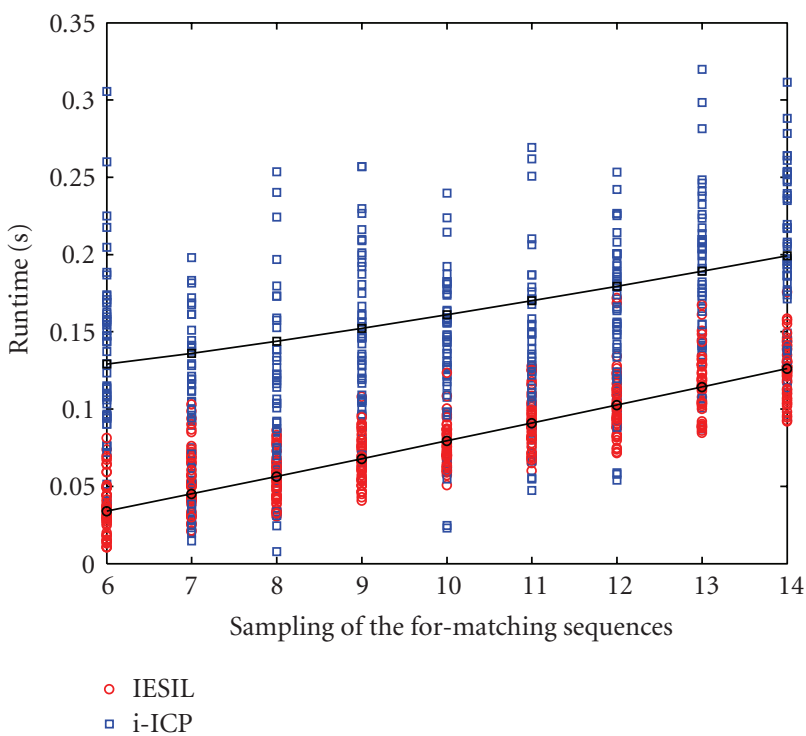

(c)

FIGURE 12: Performance comparisons: (a) positioning deviation, (b) orientating deviation, and (c) positioning run-time.

line. Besides the low-level noises during the surveying, there is a nearly constant difference about $15 \mathrm{nT}$. So, this condition with great deviation is the typical one for experiments, and this presents a more difficult testing to the new algorithm.

To test the combination algorithm's applicability to the diversity of sampling noises, the constant part of daily variation is subtracted from the measurement sequence for comparison. The resulted situation can be used to testify the algorithm in case of nonwhite noises in lowlevel. Upon that, the postprocessing experimental conditions will be separated into two cases for consideration: Status 1, the measurement sequence removed of the large constant deviation serves as the real sequence close to the normalization reference, as the instances at about 0 o'clock nearby in Figure 9; Status
2, the measurement sequence keeping the large constant deviation works as the unstable sequence in reality. The respective distributions are demonstrated in Figure 10, and the following work will be expanded on these two cases.

4.2.3. Positioning Results. In fact the new combination algorithm can overcome the influences of the large deviation, and the results of Status 1 and 2 are equivalent. The positioning results are showed in Figure 11. The deviation between the last locations output initially by INS and DGPS is $851.15 \mathrm{~m}$. The location deviation is rectified to $672.35 \mathrm{~m}$ by VS, while it is reduced to $393.63 \mathrm{~m}$ after the combination algorithm. The latter result satisfies the requirement posed in the supporting project concerning initial exploration of UMN. Based on the postprocessing on the local MMF data, INS data and GPS 
data, Hausdorff-based RC and IESIL combined algorithm have been basically validated.

The rectified deviation of $393.63 \mathrm{~m}$ is still not ideal and needs further improvements. Combined with Figure 10, it can be learnt that the distribution trend of the left sequence (Status 1) is similar with the real sequence, but obviously there are still some differences. Hence, it is not enough to just relying on algorithms to overcome the incoincidence between the sampled and real sequences, and the real solution for positioning in UMN is still to model the geomagnetic variations accurately.

\section{Discussions}

5.1. Performance Comparison. The performance comparison is based on the conditions which are identical to the simulation experiments in Section 4.1. Hausdorff-based RC is the expansion of the traditional VS algorithm without much difference in run-time, while IESIL and i-ICP both based on the isograms generate the results with almost the same accuracy. Thereupon, performance comparison can focus on some special aspects. As the producer of the combination algorithm's last results, IESIL will be assessed by comparing it with VS mainly for the accuracy of positioning and orientating. As the main time-consumer, IESIL will be compared with i-ICP briefly for the perspective of efficiency.

Different level of sampling errors commonly corresponds to different positioning accuracy, and so the positioning accuracy of the algorithm is considered along with the fault tolerance. With the noises increasing, the comparison between IESIL and VS is charted in Figures 12(a) and 12(b). Under the low-level measurement errors, positioning and orientating by IESIL have better accuracy than by VS. But with the noises increasing, IESIL's performance will get worse due to the reduction of the for-matching sequences, which is caused by the crossing-lost between the isograms and the intersection lines. VS keeps more stable both in positioning and orientating, although the results are not very satisfactory.

As indicated in Figure 12(c) IESIL is better than iICP on run-time, although it is worse than VS. It can be concluded that IESIL is a good compromise scheme, which balances both positioning accuracy and run-time. All in all, the combination algorithm is a better navigation technique compared to the traditional correlation-type algorithms and fits for the specialties of UMN.

5.2. Further Improvements. (A) The recomparison in Step 4 of IESIL can be expanded to the four vertexes of the subcell, which is determined by quartering the grid cell of previous step and takes the EE-point (solved in Step 3) on the first contour as center. Then the same recomparison process is carried out iteratively, and this can increase the algorithm's fault-tolerance.

(B) IESIL may encounter the failure of searching the intersection lines in case of large measurement errors. If IESIL finds no matching points finally, VS, which is relatively more robust under big errors shall be assumed as the alternative. Actually, IESIL, VS and i-ICP shall be programmed in parallel for cross-referencing.
(C) IESIL's performance will decrease with noises increasing, so some good noises-reducing methods are necessary. Additionally, the great discrete degree of samplings occasionally existed will increase the difficulty of noises recognition. Wavelet-based noises removal methods accompanied with multiscale analysis can be applied on the measurement sequence to ensure the new algorithm producing better results.

(D) The geomagnetic gradient measurement can reduce the influences of the daily variation and reflect the real distribution of the geomagnetic field more accurately. Hausdorff-based RC with gradients as variables will have higher accuracy.

\section{Conclusions}

This paper proposes an appropriate matching scheme and a combined positioning algorithm for UMN. Hausdorff-based RC preliminarily gives a solution for the most confusing issue caused by the geomagnetic fluctuations, such as daily variation. IESIL overcomes not only VS's inherent low accuracy caused by the assumption ofno heading error but also i-ICP's time-consuming which sources from the iterative rotation and shifting operations. The experiments based on the simulation platform and the real-surveyed data both validate the new algorithm, and the efficiency and accuracy of the combination algorithm are also relatively satisfactory. In view that the research of UMN is just in the beginning phase, the aforementioned work has given a basic verification of UMN's potentiality.

\section{Acknowledgment}

This work is supported by the National High Technology Research and Development Program of China Grant no. 2007AA09Z201.

\section{References}

[1] H. Rice, L. Mendelsohn, R. Aarons, and D. Mazzola, "Next generation marine precision navigation system," in Proceedings of the IEEE Position Location and Navigation Symposium (PLANS '00), pp. 200-206, 2000.

[2] F. Goldenberg, "Geomagnetic navigation beyond the magnetic compass," in Proceedings of the IEEE Position Location and Navigation Symposium (PLANS '06), pp. 684-694, 2006.

[3] H. Rice, S. Kelmenson, and L. Mendelsohn, "Geophysical navigation technologies and applications," in Proceedings of the IEEE Position Location and Navigation Symposium (PLANS '04), pp. 618-624, 2004.

[4] C. Teixeira and A. M. Pascoal, "Geophysical navigation of autonomous underwater vehicles using geomagnetic information," in Proceedings of the IFAC Workshop on Navigation, Guidance and Control of Underwater Vehicles (NGCUV '08), Killaloe, Canada, April 2008.

[5] L. Stutters, H. Liu, C. Tiltman, and D. J. Brown, "Navigation technologies for autonomous underwater vehicles," IEEE Transactions on Systems, Man and Cybernetics, Part C, vol. 38, no. 4, pp. 581-589, 2008. 
[6] C. Tyren, "Magnetic anomalies as a reference for groundspeed and map- matching navigation," Journal of Navigation, vol. 35, no. 2, pp. 242-254, 1982.

[7] J. L. Kenneth, M. L. Catherine, M. E. Llewellyn, A. B. Dean, and S. Timothy, "Animal behavior: geomagnetic map used in sea-turtle navigation," Nature, vol. 428, no. 6986, pp. 909-910, 2004.

[8] S. Akesson, A. C. Broderick, F. Glen, et al., "Navigation by green turtles: which strategy do displaced adults use to find Ascension Island?” Oikos, vol. 103, no. 2, pp. 363-372, 2003.

[9] L. Tian, Y. Pan, and W. Lin, "Progress in studies of geomagnetic navigation of animals," Science Bulletin in China, vol. 13, no. 1, pp. 17-19, 2005.

[10] K. P. Behzad and K. Behrooz, "Registration algorithms for geophysical maps," in Proceedings of the MTS/IEEE Oceans Conference (OCEANS '97), vol. 2, pp. 974-980, 1997.

[11] N. Kato and T. Shigetomi, "Underwater navigation for longrange autonomous underwater vehicles using geomagnetic and bathymetric information," Advanced Robotics, vol. 23, no. 7-8, pp. 787-803, 2009.

[12] I. Nygren and M. Jansson, "Terrain navigation using the correlator method," in Proceedings of the IEEE Position Location and Navigation Symposium (PLANS '04), pp. 649-657, 2004.

[13] Y. Lin, L. Yan, and Q. Tong, "Underwater geomagnetic positioning mode based on ICP algorithm," in Proceedings of the IEEE International Conference on Robotics and Biomimetics (ROBIO '07), pp. 2115-2120, 2007.

[14] Z. Ren and G. Yang, "Geomagnetic matching algorithm based on vector search," Journal of Chinese Inertial Technology, vol. 16, no. 4, pp. 424-427, 2008.

[15] J. A. Lowrey III and J. C. Shellenbarger, "Passive navigation using inertial navigation sensors and maps," Naval Engineers Journal, vol. 109, no. 3, pp. 245-251, 1997.

[16] W. R. Baker and R. W. Clem, "Terrain contour matching (TERCOM) primer," Tech. Rep. ASD-TR-77-61, Aeronautical System Division, Wright-Patterson AFB, Dayton, Ohio, USA, 1997.

[17] P. J. Besl and N. D. McKay, "A method for registration of 3D shapes," IEEE Transactions on Pattern Analysis and Machine Intelligence, vol. 14, no. 2, pp. 239-256, 1992.

[18] U. Hisashi, N. Maroka, and K. Tsuneomi, "A study of annual variations in the geomagnetic total intensity with special attention to detecting volcanomagnetic signals," Earth, Planets and Space, vol. 52, no. 2, pp. 91-103, 2000.

[19] A. S. Paul and E. A. Wan, "Dual Kalman filters for autonomous terrain aided navigation in unknown environments," in Proceedings of the International Joint Conference on Neural Networks (IJCNN '05), vol. 5, pp. 2784-2789, 2005.

[20] R. Karlsson and F. Gustafsson, "Particle filter for underwater terrain navigation," in Proceedings of the IEEE Workshop on Statistical Signal Processing, pp. 526-529, 2003. 